IEEE Transactions on Nuclear Science, Vol. NS-28, No. 3, June 1981

\title{
ADVANCED STOCHASTIC COOLING MECHANISMS
}

\author{
W. Kells \\ Fermi National Accelerator Laboratory" \\ P.0. Box 500 \\ Batavia, Illinois 60510
}

\section{Introduction}

The heart of all proposed Stochastic $\bar{p}$ accumulators is a "fast" momentum precooling section, whose performance ( $t$ ime to $c 00 l \delta p / p$ by a certain factor) must match the cycle time of the accelerators which supply and empty it of $\overline{p s}$ and/or the cooling time of a companion stacking section. Here I discuss some Inherent design limitations of the fast cooling section and some "ways out".

The usual Fokker-Planck formulation of momentum cooling admits an invariance (ideally, with no circuit noise) such that for cooling by a fixed fraction $F$ :

$$
T_{c}=K(F) \frac{N}{2 W}
$$

where $T$ is the cooling time, $N$ the total number of $\bar{p}_{s}$ being cooled, and $W$ the effective feedback channel bandwidth. The function $K(F)$ is normalized such that $\partial \mathrm{R} /\left.\partial \mathrm{F}\right|_{\mathrm{F}=1}=1$. This formulation suggests $\mathrm{a}$ wide latitude in design possibilities. Precooler circumference does not enter so that it could be a free parameter to satisfy other (than cooling) constraints. The ratio $N / T$ is constant so that either large $N$ stacks of $\bar{p} s$ or many dilute batches of ps could be precooled to give equivalent net ylelds.

In practice such a wide latitude of design is not possible. Several reasons for this are independent of the cooling itself (e.g. production target heating limitations). However the state of art of filter momentum cooling places some restrictions which exclude desirable designs. In particular, to get by with small precooler rings and/or lessen the target heating problems one would like to consider cooling many dilute $\vec{p}$ batches sequentially with Tc very small. Unfortunately the signal to noise (S/N) ratio is very small in this case while the broad band power required to lower $I_{0}$ would be prohibitive. I w1ll discuss a technical approach which avolds this dilemma and, finally, outline scenarios employing the techniques.

\section{Cavity Pick Ups}

The conventional longltudinal pickup or kicker is a beam-wall gap type cavity (or, equivalently, described as a beam transformer) loaded with ferrite to give very low $Q$ and hence large bandwidth. 2 The beam coupling impedance of such a device must be proportional to its $Q$. This fact necessitates hundreds of such devices ganged; at the P.U. end to achieve good $S / N$ and at the kicker end to reduce the final amplifier power. Technically the intervening electronics (P.U. to kicker) cannot be improved to achieve better $S / N$ or higher power, since these are incompatible with large instantaneous band width (200-500MHz).

Conceptually, one could span the same large $W$ with a P.U. consisting of a large number of higher $Q$ cavities each covering contiguous frequency bands. Indeed, this is feasible under certain conditions,

* Operated by Universities Research Association, Inc. under contract with the U.S. Department of Energy. where the Schottky signal is concentrated in non overlapping bands. Similarly the kicker may consist of identical cavities, so that the cooling circuit is made up of $M$ separate narrow band channels.
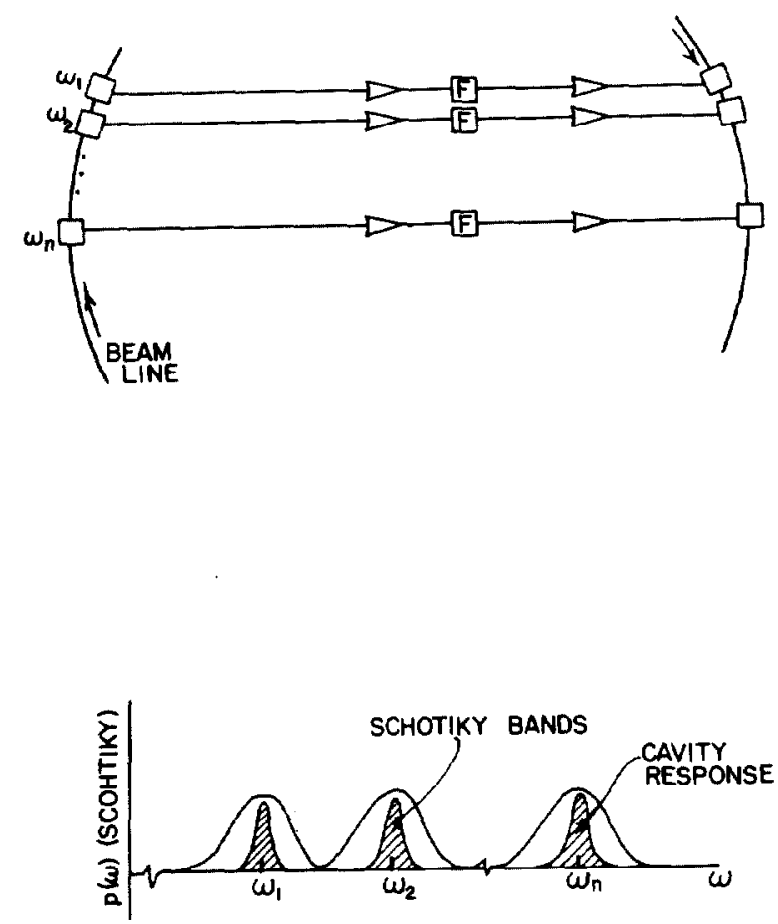

Keep in mind that Figure 1 illustrates only the simplest case, where Schottky bands and cavities are uniquely paired. For simplicity I discuss this case, although cavities spanning several bands (lower $Q$, fewer cavities) can be considered. This condition is expressed as:

$$
Q_{1} 2 w_{i} / w_{0}
$$

On the other hand, for uniform gain response throughout the Schottky band the cavity response must be signifacantly wider than the Schottky band width:

$$
\left(n^{\delta p / p}\right)^{-1} \equiv \omega_{0} / \delta \omega_{0}>Q_{1}
$$

(2) and (3) together are merely a restatement of the distinctness of all the Schottky bands, a well known criteria for the applicability of feedback cooling. 1 , 3

The response of the entire array of $M$ cavities may be examined as the sum of transient responses (to a single particle's passage) of the individuals:

$$
I(t) \propto q / Z_{s} e^{-G t} \underset{\substack{\sum_{2} \\ h_{1}}}{h_{2}} e^{-i n \omega_{0} t}
$$


Where we assume that $G \equiv w_{i} / 2 Q_{i}$ is the same for each cavity (by fabrication). In this case $G<<W^{-1}$ so the time domain F.U. signal is essentially given by the bracket; a pulse of width $(2 w)^{-1}$. In the time domain such a P.U. array is entirely equivalent to the usual P.U. By reciprocity it can be demonstrated that the kicker works as well.

But there is more! The bracket in (4) is periodic with period $2 \pi / \omega_{0}$. To avoid these after pulses:

$$
Q_{1}<\pi \omega_{i} / \omega_{0}
$$

Which must be compromised with (2). Also my assumption that $\mathrm{G} \equiv$ const. will finally (high enough W) be at odds with (3) since $Q_{i}$ must grow with frequency. In frequency domain these qualifications are equivalent to the fact that the cavities have an inherent phase shift vs. frequency (and the kicker's does not cancel the p.u.'s!) Whereas the ideal broadband system does not. In practice the case of bad mixing is usually encountered, so that for any practical W (say $5500 \mathrm{MHz}$ ), (3) can still be maintained. Unfortunately, one could probably not be able to use such p.u./k to extend practical bandwidths or to work near the good mixing limit (even not using filter cooling; see below).

The conditions on cavity $Q$ can be looked at from the point of view of beam coupling impedance. We would like to know if such an array can be more sensitive than an equivalent length of broadband couplers. In the gridded approximation,

$$
\mathrm{z}_{s}=486 \Omega \mathrm{LVQ} / \mathrm{c}
$$

For $\mathrm{L}=6 \mathrm{~cm}$ at $v=500 \mathrm{MHz}, Q=250$ (see below) $\mathrm{z}_{\mathrm{s}}=$ $12 \cdot 2 \mathrm{k} \Omega$, which is to be compared with $Z$, (\# pickups) $x$ (coupling 1mpedance per p.u.) forf the usual broadband systems (typically $z$ fo $=(100-200) \times$ $\left.(10-50 \Omega)=10^{3}-10^{4} \Omega\right)$. The above effse represents $1360 \Omega / \mathrm{m}$, compared to $420 \Omega / \mathrm{m}$ for the AA precooler p.u. ${ }^{6}$ A relevant sensitivity is relative to the overall circuit electronic noise power. In this consideration lies the particular advantage of the cavity p.u. If $Q$ $\alpha$ (wall loss resistivity) ${ }^{-1}$ The relative Johnson nolse from the cavities goes as $Q^{-1}$. Better, the $Q$ could be killed by overcoupling the signal output, in which case good copper cavities would be entirely negligible noise sources. Also, the preampliflers, one per cavity, are narrow band and can now be of entirely different technology than those available for broadband P.Us. Simple parametric amplifiers with $520^{\circ} \mathrm{K}$ N.F. could be used, with the cavity itself as the input tank. 5

The essential advantage of breaking up $w$ into many narrow bands carries over to the kickers. Here the problem is to supply enough voltage kick/turn to the particles to sustain fast cooling (rms rates 10 mev/s). Narrow band tube amplifiers can easily supply more power than the reasonable broadband devices now contemplated.

On the other hand a large number of preamps and power amplifiers are needed. For filter cooling the same number of stub lines would also be needed. Fortunately an alternative filterless cooling method is possible. ${ }^{3}$ In fact, this method is ideally suited for use with cavity array p.u./kickers since it works best with a lower noise electronic circuit. This concept in comparison with filter cooling is sketched in Figure 2.

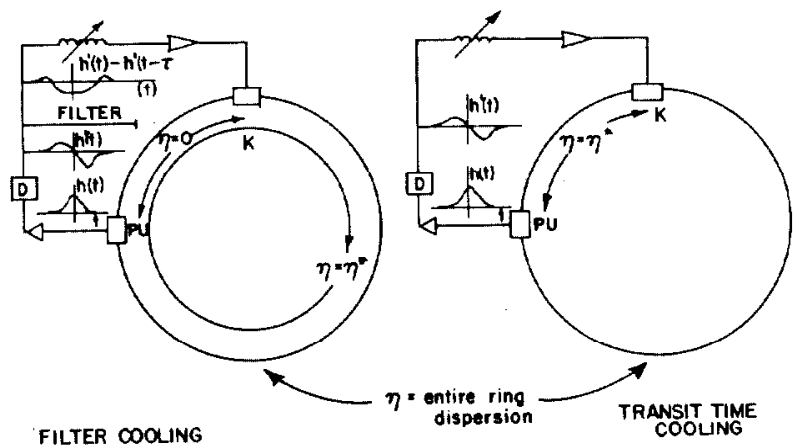

A particular interest at Fermilab for such cooling schemes stems from our accumulator designs requiring a large cooling factor and deceleration to a low energy electron cooler. Under these oircumstances it is possible to cool without shrinking the schottky bands by a large factor. This condition is also best suited to the "transit time" cooling method. ${ }^{3}$ This fact is illustrated by Fokker-Planck simulation of both cooling methods for equivalent, realistic feedback circuits (see Figure 3 ).

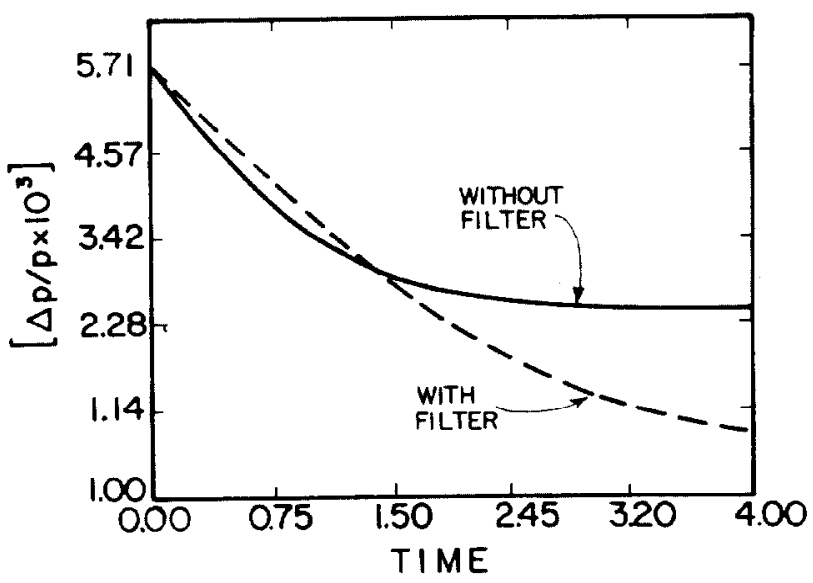

It is seen that the rates at early times are comparable but much less cooling is obtained asymptotically with the transit-time circuit (note that the same noise level broad band circult is operating for both these curves).

\section{Application}

Besides the advantages of lower noise (or same notse level but shorter p.u. net lengths) and higher attainable power levels, the above scheme (cavity p.u./kickers mated with transit time cooling) allows new accumulation scenarios to be contemplated. Since any complete, consistent scenario requires detailed consideration of many interconnected problems I will suggest only some general outlines.

It would be advantageous to construct a precooler with as small a circumference as bending magnet field allows (for the $8 \mathrm{GeV}$ top energy of Fermilab designs. This would be $1 / 4 \sim 1 / 3$ Booster C.) For noise 
insignificant we see from (1) that small rings do not effect cooling per say. Also, the formula for for required kicker power:

$$
\mathrm{P}_{\mathrm{k}} \propto \mathrm{N} \Delta \mathrm{P}^{2} \mathrm{R}^{2} / \mathrm{W} \omega_{0}
$$

where $R \equiv$ instantaneous cooling rate, shows that $P_{k}$ goes down with circumference.

The traditional objections to reducing precooler circumference are 1, that the straight section length available for PU and kicker is proportionally reduced; and 2, that a proportionally large number of batches must be sequentially extracted from the Main Ring. The latter is really an advantage as far as target heating is concerned. However, unless the cooling time per batch can be greatly reduced, such a sequential approach greatly lengthens the overall MR cycle time. The cooling technology of section II presents a way out of this dilemma.

It is feasible to incorporate smaller precoolers in two ways. First a given natural batch length (e.s. 1/13 MR for the Fermilab case) can be shortened in the proton ring by $R F$ manipulation. ${ }^{7}$ Second, the proton ring can be segmented into more batches (say $1 / 26$ or $1 / 39$ for the Fermilab MR). The cooling per batch can be in principal faster by a factor equal to the batch number ( 213 for Fermilab) since $N$ is reduced (see eg. (1)). Thus with no overhead time for precooler cycling this class of schemes could preserve net $\bar{p}$ production yield. on the other hand $P_{1}$ increases with the batch number divided by the increase in circumference. Both the rate and power increase can be achieved with the narrow band approach. The practical limitation comes from the implied increase in precooler deceleration rate. Several tentative scenarios have been devised which require only $1 / 3$ or $1 / 4$ Fermilab booster circumference precoolers to attain accumulation rates equal to the best attainable in the conventional full booster scenarios. An essential additional element in these sequential scenarios is the use of electron cooling at relatively high energy $(\Omega 2.0 \mathrm{GeV} / \mathrm{c} \overline{\mathrm{p}}$ momentum) to allevlate the deceleration (from $5.4 \mathrm{GeV} / \mathrm{c}$ ) rate problem.

\section{Acknowledgments}

The author gratefuliy acknowleges the benefit of discussions with, and suggestions and criticisms from, F. Mills, R. Huson, J. Simpson, G. Lambertson, J. BIsognano, and P.M. McIntyre.

\section{References}

1. S. van der Meer and D. Möhl, Phys. Reports, 58, No 2. 1979.

2. G. Petrucei and L. Thorndahl, ibid.

3. W. Kells, Fermilab TM 927, 1979, unpublished.

4. J. Slater, Microwave Electronios, chapter 9, McGraw Hili, 1948.

5. C. Aitchison and A. Wong, IEEE Microwave Transactions, MTT-28, 1980.

6. AA Parameter List, 9th edition, CERN Note PS/AA/80-1.

7. Fermilab High Intensity Antiproton Source, Fermilab, 1979. 